\title{
Medienbeteiligungen politischer Parteien - zugleich eine Besprechung des Urteils des Bundesverfassungsgerichts vom 12. März 2008 - 2 BvF 4/03*
}

\author{
Malte Cordes
}

Die Medienbeteiligungen politischer Parteien ${ }^{1}$ waren wiederholt Gegenstand juristischer ${ }^{2}$ und politischer ${ }^{3}$ Debatten. ${ }^{4}$ Bereits vor zweieinhalb Jahren erklärte der Niedersächsische Staatsgerichtshof, dass das Ziel, Staatsferne, Überparteilichkeit sowie Meinungsvielfalt des Rundfunks im Sinne der Rechtsprechung des Bundesverfassungsgerichts (BVerfG) zu sichern, nicht den weitgehenden Ausschluss politischer Parteien von der Veranstaltung privaten Rundfunks rechtfertige. ${ }^{5}$ Der Zweite Senat des BVerfG musste nunmehr über den Normenkontrollantrag von 232 SPD-Abgeordneten des Deutschen Bundestages gegen $\$ 6$ Abs. 2 Nr. 4 des Hessischen Privatrundfunkgesetzes (HPRG) entscheiden. Diese Vorschrift verwehrt es politischen Parteien und Wählergruppen, sich direkt oder mittelbar an privaten Rundfunkunternehmen zu beteiligen.

Gegenwärtig bestehen Beteiligungen aller im 16. Bundestag vertretenen Parteien an Medienunternehmen. ${ }^{6}$ Insbesondere die Deutsche Druck- und Verlagsgesellschaft mbH

* Die Entscheidung ist mit Gründen veröffentlicht in: NVwZ 2008, S. 658 ff.

1 Vgl. zur allgemeinen erwerbswirtschaftlichen Betätigung politischer Parteien Peter M. Huber, Parteien in der Demokratie, in: Peter Badura / Horst Dreier (Hrsg.), Festschrift 50 Jahre Bundesverfassungsgericht, Bd. 2, Tübingen 2001, S. 609 ff., S. 619 f.; Peter M. Huber, Medienbeteiligungen politischer Parteien, in: K\&R 2004, S. 216 ff., S. 219. Anderer Ansicht Hans-Peter Schneider, Parteifinanzen und Parteivermögen, in: Helga Grebing / Karin Junker (Hrsg.), Frau. Macht. Zukunft, Marburg 2001, S. 325 ff., S. 326 ff.

2 Vgl. Miroslav Angelov, Vermögensbildung und unternehmerische Tätigkeit politischer Parteien, Berlin 2006; Miroslav Angelov / Alexander Roth, Auf dem Weg zu mehr Transparenz bei den Einnahmen politischer Parteien aus Wirtschaftstätigkeit, in: KritV 2006, S. 21 ff.; Peter M. Huber, a.a.O. (Fn. 1), S. 216 ff., S. 609 ff.; Martin Morlok / Ulrich von Alemann / Thilo Streit (Hrsg.), Medienbeteiligungen politischer Parteien, Baden-Baden 2004 (gefördert von der dd_vg.); Markus Möstl, Politische Parteien als Medienunternehmer, in: DÖV 2003, S. 106 ff.; Alexandra Schindler, Die Partei als Unternehmer, Baden-Baden 2006, mit Überlegungen zur Übertragung des Nebentätigkeitsprivilegs auf Parteien; Tobias Schneider, Vermögen und erwerbswirtschaftliche Betätigung politischer Parteien, Hamburg 2008; Hendrik Reffken, Politische Parteien und ihre Beteiligungen an Medienunternehmen, Baden-Baden 2007.

3 LT BW-Drs. 13/1550, \$13 Abs. 3 Nr. 6 LMG BW; BayLT-Drs. 14/12033, \$ 24 Abs. 3 BayMG; HessLT-Drs. 15/1446 und 15/2200, \$ 6 Abs. 2 Nr. 4 HPRG; NdsLT-Drs. 15/450, \$ 6 Abs. 3 S. 2 - 4 NdsMG. Vgl. auch LG Berlin, in: ZUM-RD 2004, S. 129 zum Unterlassungsanspruch der SPD wegen unwahrer Tatsachenbehauptung zu ihrer Pressemacht in einer im Internet veröffentlichten Presseerklärung der Bayerischen Staatskanzlei.

4 Siehe dazu jüngst Malte Cordes, Medienbeteiligungen politischer Parteien: Zur verfassungsrechtlichen Zulässigkeit von Unternehmensbeteiligungen politischer Parteien in Presse, Rundfunk und Neuen Medien, Göttingen 2009.

5 Urteil des Niedersächsischen Staatsgerichtshofs vom 6. September 2005, Az.: StGH 4/04. Die Entscheidung ist mit Gründen veröffentlicht in: DVBl 2005, H. 23, S. 1515 ff.

6 Vgl. BT-Drs.16/5090, S. 37, S. 88, S. 185, S. 227. Eine Ausnahme stellt die CSU dar, die lediglich Verlegerin ihrer traditionellen Parteizeitung Bayernkurier ist, ebenda, S. 157. Die Grünen sind in geringem Umfang an der taz-Genossenschaft beteiligt, gemäß $₫ 24$ Abs. 7 Nr. 1 S. 3 PartG in Verbindung mit $\$ 271$ Abs. 1 S. 4 HGB nicht veröffentlichungspflichtig. 
(dd_vg.), die Unternehmensholding der SPD, ist an zahlreichen Verlagshäusern beteiligt, die wiederum Anteile an privaten Rundfunksendern halten. ${ }^{7}$ Der durchgerechnete mittelbare dd_vg.-Anteil ${ }^{8}$ liegt nach eigenen Angaben - ohne Berücksichtigung der privaten Rundfunksender in Nordrhein-Westfalen - zwischen 0,4 und 12,8 Prozent. Die einzige Direktbeteiligung der dd_vg. ist an der RPR Rheinland-Pfälzischer Rundfunk GmbH \& Co. KG („RPR 1“, „bigFM“) mit 9,2 Prozent gegeben.?

Da das Recht auf freie Meinungsäußerung geradezu ihr Lebenselixier ist ${ }^{10}$, garantiert Art. 21 Abs. 1 Satz 1 GG den Parteien die Befugnis zu aktiver Mitwirkung an der politischen Willensbildung des Volkes und macht sie ihnen zugleich zur Pflicht. ${ }^{11}$ Aus diesem Blickwinkel spricht zunächst alles für eine umfassende Medienveranstalterfreiheit der Parteien, zumal Art. 5 Abs. 1 Satz 2 GG nach ständiger Rechtsprechung des BVerfG einen Auftrag zur Gewährleistung der Rundfunkfreiheit enthält. Dieser zielt auf eine Ordnung, die insbesondere sicherstellt, dass die Vielfalt der bestehenden Meinungen im Rundfunk in möglichster Breite und Vollständigkeit Ausdruck findet. ${ }^{12}$ Hiernach würde die Inkompatibilitätsregelung des $\$ 6$ Abs. 2 Nr. 4 HPRG in die Rundfunkfreiheit der Parteien nach Art. 5 Abs. 1 Satz 2 Var. 2 GG in Verbindung mit Art. 19 Abs. 3 GG eingreifen. Hingegen sollen die Medien eine unabhängige politische Willensbildung des Volkes ermöglichen und sicherstellen. Außerdem gehört die Kontrolle staatlicher Machtausübung zu ihren Aufgaben. Aus diesem Grunde ist eine staatsunabhängige Stellung und Finanzierung der Medien erforderlich. ${ }^{13}$ Häufig werden diese deshalb neben Legislative, Exekutive und Judikative als „vierte Gewalt"14 aufgezählt. Die Gesamtschau der unterschiedlichen Funktionen von Parteien und Medien zeigt, warum Kritiker in den Medienbeteiligungen politischer Parteien einen klaren Verstoß gegen den Geist der Verfassung und das Prinzip der Gewaltenteilung sehen.

7 Amtsgericht Berlin-Charlottenburg, HRB 83297; Geschäftsbericht 2007 der dd_vg., S. 10.

8 Allerdings ist die Berechnung einer anteiligen Auflage oder Hördauer methodisch ungeeignet, die Auswirkungen der Medienbeteiligungen von Parteien auf die Vermittlungs- und Kontrollfunktion der Medien richtig darzustellen. Eine Beeinflussung wirkt sich immer auf die Gesamtauflage oder das Gesamtprogramm aus.

9 Vgl. Überblick über Rundfunkbeteiligungen http://www.ddvg.de/ausgewaehltethemen/ueberblick_ueber_rundfunkbeteiligungen.pdf (Abruf: 15. September 2008), auch zum lizenzrechtlichen Sonderfall in Nordrhein-Westfalen. Dort ist die dd_vg. in teilweise erheblich größerem Umfang mittelbar an einigen Betriebsgesellschaften beteiligt.

10 Wilhelm Henke, Das Recht der politischen Parteien, Göttingen 1972, S. 239.

11 Hans H. Klein, Verfassungsrechtliche Erwägungen, in: Orientierungen zur Wirtschafts- und Gesellschaftspolitik 2004, H. 3, S. 68 ff., S. 70.

12 BVerfGE 57, S. 295, S. 319; BVerfGE 73, S. 118, 152 f.; BVerfGE 90, S. 60, S. 88; BVerfGE 114, S. 371, S. 387 ff.; zuletzt BVerfG, in: NVwZ 2007, S. 1287 ff., ständige Rechtsprechung.

13 Frank Fechner, Medienrecht, Tübingen 2007, Rn. 26, 41.

14 Kritisch dazu Martin Löffler / Reinhart Ricker, Handbuch des Presserechts, München 2005, 3. Kap. Rn. 25. Bei Herbert Bethge, in: Michael Sachs (Hrsg.), Grundgesetz. Kommentar, München 2007, Art. 5 Rn. 66 wird die Zuschreibung der Medien als vierte Gewalt als „soziologisches Wichtigkeitsattribut" (nach Rupert Scholz) gekennzeichnet; Rudolf Streinz, Der Einfluss der Verfassungsrechtsprechung auf die Pressefreiheit, in: AfP 1997, S. 857 ff., S. 868 f. bezeichnet diese als faktisch, nicht rechtlich. 


\section{Verfahrenshintergrund}

Wer Rundfunk veranstalten will, bedarf nach dem Hessischen Privatrundfunkgesetz einer Zulassung. Sie wird auf schriftlichen Antrag durch die Hessische Landesanstalt für privaten Rundfunk und neue Medien erteilt. Gemäß $\$ 6$ Abs. 2 Nr. 4 HPRG darf die Zulassung an Parteien oder Wählergruppen sowie an Unternehmen und Vereinigungen, an denen Parteien oder Wählergruppen beteiligt sind, nicht erteilt werden. Gleiches gilt für Treuhandverhältnisse. Von der Neuregelung betroffen war der Radiosender „Hit Radio FFH“, an dem die dd_vg. durch verschiedene Unternehmensbeteiligungen, insbesondere über die Klaus Lage GbR, mittelbar Anteile in Höhe von 2,34 Prozent hielt. Auf entsprechende Aufforderung der Landesmedienanstalt gab die dd_vg. ihre Beteiligung an diesem Radiosender auf. ${ }^{15}$

\section{Entscheidung des Bundesverfassungsgerichts}

Der Zweite Senat des BVerfG stellte mit Urteil vom 12. März 2008 fest, dass die angegriffene Norm mit Art. 5 Abs. 1 Satz 2 GG in Verbindung mit Art. 21 Abs. 1 GG unvereinbar sei. Dem Gesetzgeber stehe es zwar frei, Parteien die unmittelbare oder mittelbare Beteiligung an privaten Rundfunkunternehmen insoweit zu untersagen, als sie dadurch bestimmenden Einfluss auf die Programmgestaltung oder die Programminhalte nehmen könnten. Das absolute Verbot für Parteien, sich an privaten Rundfunkveranstaltungen zu beteiligen, sei dagegen mit der Verfassung nicht vereinbar. Der Gesetzgeber ist gehalten, bis zum 30. Juni 2009 den Verfassungsverstoß durch eine Neuregelung zu beheben. Die Entscheidung erging zur materiellen Verfassungsmäßigkeit des $\$ 6$ Abs. 2 Nr. 4 HPRG mit fünf zu drei Stimmen, im Übrigen einstimmig.

\section{Kritische Würdigung}

\subsection{Formelle Verfassungsmäßigkeit}

Art. 21 Abs. 3 GG stellt einen Gesetzgebungsauftrag ${ }^{16}$ an den Bund zur Ausgestaltung des Parteienrechts dar. Er begründet eine ausschließliche Bundeskompetenz. ${ }^{17}$ Eine Begrenzung des sachlichen Umfangs der Gesetzgebungskompetenz erfolgt durch den Bezug des „Nähe-

15 Allerdings gab die Verlagsgesellschaft Madsack GmbH \& Co. KG ihre indirekte Beteiligung an der Radio/Tele FFH GmbH \& Co. BetriebsKG einfach an die Beteiligungsgesellschaft Neue Zeitung $\mathrm{mbH}$ ab, deren Alleineigentümerin sie ist, vgl. SPD-Beteiligungstrick, in: Focus vom 25. Februar 2002, S. 12.

16 Ähnlich Wilhelm Henke, in: Rudolf Dolzer / Christian Waldhoff/ Karin Graßhof(Hrsg.), Bonner Kommentar zum Grundgesetz, Heidelberg 1991, Art. 21 Rn. 376 („Regelungsauftrag“); ebenso das Bundesverfassungsgericht in der vorliegenden Entscheidung.

17 Rudolf Streinz, in: Hermann von Mangoldt / Friedrich Klein / Christian Starck (Hrsg.), Das Bonner Grundgesetz. Kommentar, München 2005, Art. 21 Rn. 253 mit weiteren Nachweisen; vgl. BVerfGE 3, S. 383, S. 404; BVerfGE 24, S. 300, S. 354; BVerfGE 41, S. 399, S. 425. Umfassend Angelika Harms, Die Gesetzgebungszuständigkeit des Bundes aus Art. 21 Abs. 3 GG in Abgrenzung zum Zuständigkeitsbereich der Länder, Frankfurt am Main 1986, S. 86 ff. 
ren" auf die vorhergehenden Absätze, also die in Art. 21 Abs. 1 und 2 GG statuierten Inhalte, die allerdings weitreichend sind. ${ }^{18}$ Gleichwohl gilt, dass nicht immer und überall dort, wo auch Parteien tätig werden, eine ausschließliche Gesetzgebungszuständigkeit des Bundes gegeben ist. ${ }^{19}$ Bei Überschneidungen mit anderen Gesetzgebungszuständigkeiten des Bundes oder der Länder ist im Zweifel der Schwerpunkt der gesetzlichen Neuregelung entscheidend. ${ }^{20}$

Das BVerfG ist der Auffassung, dass der maßgebliche Normzweck der Regelung des $\$ 6$ Abs. 2 Nr. 4 HPRG nicht für einen parteienrechtlichen, sondern für einen rundfunkrechtlichen Schwerpunkt der Regelung spreche. Hinzu komme, dass in der Staatspraxis die Thematik der beanstandeten Regelung nicht im Parteiengesetz aufgegriffen, sondern dem Rundfunkrecht zugeordnet worden sei. ${ }^{21}$

Bei der Auslegung von Kompetenzbestimmungen wird der Staatspraxis „besonderes Gewicht“ zuerkannt. ${ }^{22}$ Keine Bedeutung dürfte indes einer verfassungswidrigen Staatspraxis zukommen. ${ }^{23}$ Bislang war ohnehin lediglich die Neuregelung des Niedersächsischen Landesmediengesetzes Gegenstand eines verfassungsgerichtlichen Verfahrens, das sich mit einem (relativen) Verbot für Parteien, sich an privaten Rundfunkveranstaltungen zu beteiligen, auseinandersetzte. Ferner gingen sowohl der CDU/CSU-Gesetzentwurf in der 14. Wahlperiode $^{24}$ als auch der FDP-Gesetzentwurf in der 15. Wahlperiode des Bundestages ${ }^{25}$ von einer ausschließlichen Bundeskompetenz aus. $\$ 24$ Abs. 1 Satz 2 in Verbindung mit Abs. 6 Nr. 1 A. II. 1. des Parteiengesetzes (PartG), wonach Parteien ihre Beteiligungen an Unternehmen im Rechenschaftsbericht offenlegen müssen, stellt zwar allgemein auf die Transparenz dieser Beteiligungen $\mathrm{ab}^{26}$, jedoch wird auch die Benennung der Hauptprodukte von Medienunternehmen nach $\$ 24$ Abs. 7 Nr. 2 PartG verlangt, soweit Beteiligungen an diesen bestehen. Auch der interfraktionelle Gesetzentwurf zur Novellierung des Parteiengesetzes 2002 übersah die Besonderheiten der Medienbeteiligungen von Parteien nicht:

18 Martin Morlok, in: Horst Dreier (Hrsg.), Grundgesetz. Kommentar, Tübingen 2006, Bd. 2, Art. 21 Rn. 161 kennzeichnet die Grundlagen des Parteienrechts in erheblicher Breite (,Sonderrecht der Parteien“); zuvor Gerd Roellecke, in: Dieter C. Umbach / Thomas Clemens (Hrsg.), Grundgesetz. Mitarbeiterkommentar und Handbuch, Heidelberg 2002, Bd. 1, Art. 21 Rn. 60; Rudolf Streinz, a.a.O. (Fn. 16), Art. 21 Rn. 254.

19 Christoph Gusy, in: Erhard Denninger / Wolfgang Hoffmann-Riem / Hans-Peter Schneider / Ekkehart Stein (Hrsg.), Kommentar zum Grundgesetz für die Bundesrepublik Deutschland, Neuwied 2001, Art. 21 Rn. 144.

20 Ebenda; Bodo Pieroth, in: Hans D. Jarass / Bodo Pieroth, Grundgesetz für die Bundesrepublik Deutschland. Kommentar, München 2007, Art. 70 Rn. 7.

21 Vgl. mit ähnlicher Begründung StGH Niedersachsen, a.a.O. (Fn. 4), S. 1516. Kritisch Joachim Wieland, Medienbeteiligung politischer Parteien, in: Martin Morlok / Ulrich von Alemann / Thilo Streit, a.a.O. (Fn. 2), S. 103 ff., S. 112 (Einzelfallgesetz).

22 BVerfGE 68, S. 319, S. 328; BVerfGE 106, S. 62, S. 105; BVerfGE 109, S. 190, S. 213.

23 Bodo Pieroth, a.a.O. (Fn. 19), Art. 70 Rn. 6.

24 BT-Drs. 14/7441, S. 3; zustimmend Peter M. Huber, a.a.O. (Fn. 1), S. 222. Der Gesetzentwurf war erfolglos, da die SPD im Gegenzug wohl mit einem Verbot der Spenden juristischer Personen drohte, Hans H. Klein, Medienbeteiligung politischer Parteien, in: Martin Morlok / Ulrich von Alemann / Thilo Streit, a.a.O. (Fn. 2), S. 77 ff., S. 81.

25 BT-Drs. 15/3097, S. 3. Auch dieser Gesetzentwurf scheiterte an der rot-grünen Bundestagsmehrheit.

26 Burkhard Küstermann, Das Transparenzgebot des Art. 21 Abs. 1 S. 4 GG und seine Ausgestaltung durch das Parteiengesetz, Göttingen 2003, S. 191 f. 
„Betreibt eine Partei Medienunternehmen oder hält sie an diesen Anteile, muss sie in Zukunft die Namen veröffentlichen, die die Produkte dieser Beteiligungen tragen. Dies soll die Öffentlichkeit auf die Möglichkeit einer parteipolitischen Einflussnahme auf den redaktionellen Inhalt des Mediums hinweisen. "27 Die Auflösung dieser strukturellen Kollision und Spannung verortete der Gesetzgeber mithin im Parteienrecht des Bundes und nicht im Presse- oder Rundfunkrecht der Länder. Eine parteienrechtliche Inkompatibilitätsregelung ist auch deshalb angezeigt, weil sie sich sowohl auf den Presse- als auch den Rundfunkbereich und auf die Neuen Medien ${ }^{28}$ erstrecken würde. Schließlich spricht für die Annahme einer ausschließlichen Gesetzgebungszuständigkeit des Bundes, dass aufgrund der strukturellen Inkompatibilität auch der grundgesetzliche Mitwirkungsauftrag der Parteien nach Art. 21 Abs. 1 Satz 1 GG gefährdet ist. Zudem fallen Bestimmungen, mit denen der Wirkungskreis der Parteien in ihrer Vermittlungsfunktion zwischen Volk und Staatsorganen ausgestaltet wird, nach den Ausführungen des Bundesverfassungsgerichts in die Gesetzgebungskompetenz des Art. 21 Abs. 3 GG.

Die wirtschaftliche Betroffenheit der Parteien ist - entgegen der Auffassung der Antragsgegner - auch nicht nur eine insoweit nicht maßgebliche Nebenfolge der Regelung. Das zeigt zum Beispiel der Umstand, dass die Unternehmensholding der SPD im Geschäftsjahr 2007 einen Jahresüberschuss in Höhe von 17,2 Mio. € erzielte. Davon profitierte auch die SPD als Gesellschafterin der dd_vg. ${ }^{29}$ Aus dem Bilanzgewinn des Jahres 2006 wurden 2007 insgesamt 8,7 Mio. $€$ ausgeschüttet (Vorjahr: 7,3 Mio. €). Gleichzeitig verbesserte das Unternehmen seine Eigenkapitalbasis. ${ }^{30}$ Hiernach ist zumindest die latente Gefahr einer Störung der für den demokratischen Meinungs- und Willensbildungsprozess notwendigen Verbindung zwischen den Parteien und ihrer Wählerschaft gegeben. ${ }^{31}$ Sie gewinnt durch den Umfang der (teilweisen) staatlichen Parteienfinanzierung, wonach die Parteien Gefahr laufen, sich aus ihrer gesellschaftlichen Verwurzelung zu lösen, erheblich an Gewicht. ${ }^{32}$ Eine Rückkopplung an das Volk als den eigentlichen Träger der Staatsgewalt ist nicht mehr gewährleistet. ${ }^{33}$ Außerdem argumentiert die SPD, dass ihre Einnahmen aus Unternehmenstätigkeit und Beteiligungen gemessen an ihren Gesamteinnahmen lediglich einen Bruchteil

27 BT-Drs. 14/8778, S. 19.

28 Die Internetangebote der Parteien werden offen als Instrument der unmittelbaren Meinungsverbreitung eingesetzt. Überdies ist eine Alleinanbieterstellung für politische Meinungen im Internet undenkbar, so dass lediglich die Abgrenzung zu den anderen Medienfreiheiten problematisch ist. Dazu Helmuth Schulze-Fielitz, in: Horst Dreier (Hrsg.), Grundgesetz. Kommentar, Bd. 1, Tübingen 2004, Art. 5 Abs. 1, 2 Rn. 91. Allgemein zur Nutzung des Internets bei SPD und CDU Andrea Römmele, www.parteien.de, in: Helga Grebing / Karin Junker, a.a.O. (Fn. 1), S. 287 ff.

29 Die Generaltreuhänderin Barbara Hendricks, seit 2007 Bundesschatzmeisterin der SPD, hält 94,67 Prozent an der dd_vg., 5,33 Prozent werden aus steuerrechtlichen Gründen von der Solidarität GmbH gehalten, treuhänderisch für den Parteivorstand der SPD, Geschäftsbericht 2007 der dd_vg, S. 26.

30 Ebenda, S. 6.

31 Vgl. die Äußerungen von Peter M. Huber, Ulrich Karpen und Norbert Röttgen in der öffentlichen Sachverständigenanhörung der CDU/CSU-Bundestagsfraktion am 20. Oktober 2000 zum Thema „Das Transparenzgebot des Grundgesetzes und die SPD-Parteifinanzen“.

32 BVerfGE 85, S. 264, S. 287; vgl. BVerfGE 73, S. 40, S. 88. Allgemein Gregor Stricker, Der Parteienfinanzierungsstaat, Baden-Baden 1998. Kritisch Miroslav Angelov, a.a.O. (Fn. 2), S. 222 f.

33 Vgl. zur fehlenden Rückkopplung als aktuelles Krisensymptom des Parteienstaats Peter M. Huber, Der Parteienstaat als Kern des politischen Systems, in: JZ 1984, S. 689 ff., S. 692 f. 
darstellten. ${ }^{34}$ Warum aber sollte eine solche geringfügige erwerbswirtschaftliche Betätigung der Partei, die nicht unmittelbar dem grundgesetzlichen Mitwirkungsauftrag des Art. 21 Abs. 1 Satz 1 GG dient, Voraussetzung zur Erfüllung ihrer Aufgaben nach $₫ 1$ Abs. 2 PartG sein? ${ }^{25}$ Sofern die SPD dagegen über ihre Unternehmenstätigkeit und Beteiligungen gemäß Art. 21 Abs. 1 Satz 1 GG an der politischen Willensbildung des Volkes mitwirkt, würde sie entgegen anderslautenden Beteuerungen eine Einflussnahme auf die Gestaltung der öffentlichen Meinung einräumen müssen.

\subsection{Materielle Verfassungsmäßigkeit}

Grundsätzlich ist dem BVerfG zuzustimmen, dass es dem Gesetzgeber frei steht, Parteien die unmittelbare oder mittelbare Beteiligung an privaten Rundfunkunternehmen insoweit zu untersagen, als sie dadurch bestimmenden Einfluss auf die Programmgestaltung oder die Programminhalte nehmen können (vgl. $\$ 17$ des Aktiengesetzes). Dagegen ist die weitergehende Begründung, wonach ein absolutes Verbot für Parteien, sich an privaten Rundfunkveranstaltungen zu beteiligen, verfassungswidrig sein soll, Bedenken ausgesetzt.

\subsubsection{Grundsatz der Staatsfreiheit des Rundfunks}

Folgt man dem BVerfG in seinem Verständnis der Rundfunkfreiheit als dienender Freiheit $^{36}$, ist die Feststellung des Gerichts zutreffend, der Grundsatz der Staatsfreiheit des Rundfunks sei auch im Verhältnis zu den Parteien zu beachten. Zwar seien diese nicht dem Staat zuzuordnen, jedoch bestehe eine gewisse Staatsnähe der Parteien, die eine Beachtung des Grundsatzes der Staatsfreiheit des Rundfunks für die Ausgestaltung von Parteibeteiligungen an Rundfunkveranstaltern notwendig mache.

\section{Verfassungsrechtliche Stellung der Parteien}

Die verfassungsrechtliche Stellung der Parteien ist durch einen Status der Freiheit, Gleichheit und Öffentlichkeit gekennzeichnet, einer Freiheit gerade auch vom Staat, ohne deren Gewährleistung die Parteien nicht in der Lage wären, die ihnen vom Grundgesetz verbürgte öffentliche Aufgabe zu erfüllen. Hieraus ergibt sich „das Verbot jeder staatlich-institutio-

34 Die SPD nahm nach ihrer Einnahmen- und Ausgabenrechnung 2005 7.023.034€ (4,15 Prozent) über Einnahmen aus Unternehmenstätigkeit und Beteiligungen ein, BT-Drs. 16/5090, S. 61.

35 So aber Hans-Peter Schneider, a.a.O. (Fn. 1), S. 326 f.

36 Anderer Ansicht Peter Charissé, Die Rundfunkveranstaltungsfreiheit und das Zulassungsregime der Rundfunk- und Mediengesetze, Frankfurt am Main 1999, S. 148, S. 214 f.; Hubertus Gersdorf, Funktionen der Gemeinschaftsgrundrechte im Lichte des Solange II-Beschlusses des Bundesverfassungsgerichts, in: AöR 1994, S. 400 ff., S. 416; Roman Herzog, in: Theodor Maunz I Günter Dürig (Hrsg.), Grundgesetz. Kommentar, München 1989, Art. 5 Abs. 1, 2 Rn. 236; Peter M. Huber, a.a.O. (Fn. 1), S. 218; Rupert Scholz, Medienfreiheit und Publikumsfreiheit, in: Studienkreis für Presserecht und Pressefreiheit (Hrsg.), München 1980, S. 355 ff.; Christian Starck, Zur notwendigen Neuordnung des Rundfunks, in: NJW 1980, S. 1359 ff., S. 1360; ders., "Grundversorgung" und Rundfunkfreiheit, in: NJW 1992, S. 3257 ff., S. 3261 f. 
nellen Verfestigung der Parteien, insbesondere das Verbot einer Einfügung der Parteien in die organisierte Staatlichkeit “37. Besonders wichtiger Beleg für die Verankerung der Parteien in der Gesellschaft ist neben ihrer privatrechtlichen Organisationsform ${ }^{38}$ die in Art. 21 Abs. 1 Satz 3 GG enthaltene Forderung nach innerparteilicher Demokratie, die wegen der zentralen Funktion, die die Parteien innerhalb der repräsentativen Demokratie ausüben, in Verbindung mit Art. 20 Abs. 1 und 2 GG gesehen werden muss. ${ }^{39}$ Ausgangspunkt der verfassungsrechtlichen Untersuchung der Medienbeteiligungen politischer Parteien ist daher die Feststellung, dass die Parteien als „frei gebildete, im gesellschaftlich-politischen Bereich wurzelnde Gruppen " 40 in erster Linie der gesellschaftlichen und nicht der staatlichen Sphäre zuzuordnen sind. Die Parteien sind mithin Grundrechtsträger, so dass auch die Medienfreiheiten - insbesondere die Presse- und Rundfunkfreiheit gemäß Art. 5 Abs. 1 Satz 2 Var. 1 und 2 GG - nach Maßgabe von Art. 19 Abs. 3 GG auf die Parteien anwendbar sind. ${ }^{41}$ Dabei ist die grundrechtliche Freiheit der Vereinigung Konsequenz der grundrechtlichen Freiheit ihrer Mitglieder. ${ }^{42}$ Hilfreich ist die Grundrechtsberechtigung auch im Hinblick auf das Verfassungsprozessrecht, weil Art. 21 GG im Katalog der verfassungsbeschwerdefähigen Rechte des Art. 93 Abs. 1 Nr. 4a GG nicht aufgeführt ist. Sofern die Parteien aber Bürgerverbände sind, gewinnt der Einwand der SPD an Bedeutung, ihre Beteiligungen im Rundfunkbereich seien lediglich eine gewöhnliche Finanzinvestition. Denn warum sollten solche Verbände an einer erwerbswirtschaftlichen Betätigung gehindert werden?

\section{Vermittlungs- und Kontrollfunktion der Medien}

Die Information der Bürger über den Staat, ihre „wissende Nähe“, ist sowohl Voraussetzung demokratischer Wahl als auch rechtsstaatlicher Kontrolle. ${ }^{43}$ Vermittlungsfunktion bezeichnet insoweit die zentrale Aufgabe der Medien in der repräsentativen Demokratie, die in der Gesellschaft und ihren Gruppen sich unaufhörlich neu bildenden Meinungen und Forderungen kritisch zusammenzufassen, sie zur Erörterung zu stellen und an die politisch handelnden Staatsorgane heranzutragen, die auf diese Weise ihre Entscheidungen auch in

37 Konrad Hesse, Die verfassungsrechtliche Stellung der Parteien im modernen Staat, in: VVDStRL 1959, S. 48 ff., S. 51; treffend Max G. Lange, Politische Soziologie, Frankfurt am Main 1964, S. 63, „Mittel und Mittler politischer Herrschaft“.

38 Die Parteien sind mehrheitlich als nichtrechtsfähige Vereine organisiert: CDU, SPD, Die Linke und Grüne sind nicht eingetragene Vereine, dagegen sind CSU und FDP und mehrere Landesverbände der FDP eingetragene Vereine, vgl. $\$ 2$ S. 1 der Satzung der CSU; $\$ 29$ Abs. 1 der Bundessatzung der FDP.

39 Wiebke Wietschel, Der Parteibegriff, Baden-Baden 1996, S. 45.

40 BVerfGE 20, S. 56, S. 101 unter Hinweis auf BVerfGE 1, S. 208, S. 224; BVerfGE 3, S. 383, S. 393.

41 Philip Kunig, in: Josef Isensee / Paul Kirchhof(Hrsg.), Handbuch des Staatsrechts der Bundesrepublik Deutschland, Bd. 3, Heidelberg 2005, $\$ 40$ Rn. 92 (Modifikation durch Art. 21 Abs. 1 Satz 1 GG); anderer Ansicht Peter M. Huber, a.a.O. (Fn. 1), S. 220 f., S. 623 f., Art. 21 GG als abschließende Funktionsgarantie; anders noch ders, in: Hermann von Mangoldt / Hans H. Klein I Christian Starck, a.a.O. (Fn. 16), München 1999, Art. 19 Abs. 3 Rn. 259. Umfassend Wilhelm Henke, a.a.O. (Fn. 9), S. $229 \mathrm{ff}$.

42 Hans H. Klein, a.a.O. (Fn. 10), S. 70.

43 Walter Schmitt-Glaeser, Politik und Medien - ein brisantes Mischsystem, in: PM 2002, S. 11 ff., S. 13. 
Einzelfragen der Tagespolitik ständig am Maßstab der im Volk tatsächlich vertretenen Auffassungen messen können. ${ }^{44}$ Kontrollfunktion umschreibt die gerade unter den Bedingungen der Massendemokratie und der aktuellen Krisensymptome der Parteiendemokratie ${ }^{45}$ unerlässliche Aufgabe der Kritik und Kontrolle staatlicher Machtausübung. ${ }^{46}$ Der Europäische Gerichtshof für Menschenrechte spricht insoweit von den Medien als Wächter der Öffentlichkeit. ${ }^{47}$ Allerdings sind die Vermittlungsfunktionen, die die Medien als Verbindungsorgan zwischen Volk und Politik und die die Parteien „als Träger und Mittler eines freien und offenen Willensbildungsprozesses “ 48 zwischen Volk und Staat wahrnehmen sollen, nicht identisch. Vielmehr ist die politische Willensbildung des Volkes, an der die Parteien nach Art. 21 Abs. 1 Satz 1 GG mitwirken sollen, Gegenstand von Berichterstattung. 49

\subsubsection{Abhängige und herrschende Unternehmen}

Keinen Bedenken ist daher die Feststellung des BVerfG ausgesetzt, dass der Gesetzgeber von seiner Ausgestaltungsbefugnis jedenfalls dann in zulässiger Weise Gebrauch macht, wenn er den Parteien die unmittelbare oder mittelbare Beteiligung an privaten Rundfunkunternehmen insoweit untersagt, als sie dadurch bestimmenden Einfluss auf die Programmgestaltung oder die Programminhalte nehmen können.

\section{Strukturelle Funktionsstörung}

Allerdings kann gegen eine Berücksichtigung der grundrechtlichen Position der Parteien bei der Zulassung zum Privatrundfunk nach der Begründung des BVerfG nicht geltend gemacht werden, dass eine strukturelle Unvereinbarkeit von Parteien und Rundfunk bestehe. Zwar komme dem Rundfunk eine besondere Kontrollfunktion gegenüber staatlichem Handeln zu, doch sei diese lediglich eine der vielfältigen Aufgaben des Rundfunks; sie sei zudem im Bereich des privaten Rundfunks nicht von jedem Rundfunkunternehmen gleichermaßen zu gewährleisten. ${ }^{50}$ Schließlich sei auch die Funktion des Rundfunks nicht dadurch

44 So ausdrücklich BVerfGE 20, S. 162, S. 175 für die Presse.

45 Vgl. Peter M. Huber, a.a.O. (Fn. 32), S. 692 ff., der aber den Begriff „Parteienstaat“ gebraucht. Dazu Richard Stöss, Parteienstaat oder Parteiendemokratie?, in: Oscar W. Gabriel / Oskar Niedermayer / Richard Stöss (Hrsg.), Parteiendemokratie in Deutschland, Opladen 1997, S. $13 \mathrm{ff}$.

46 Markus Möstl, a.a.O. (Fn. 2), S. 107 f.

47 EuGHMR, in: EuGRZ 1995, S. 16 ff., S. 20.

48 Konrad Hesse, a.a.O. (Fn. 36), LS II. 2, S. 50. Soweit Markus Möstl, a.a.O. (Fn. 2), S. 110 von „Transmissionsriemen“ spricht, dürfte dieser Begriff nicht richtig gewählt sein, da den Parteien die Aufgabe zukommt, die auf die politische Macht und ihre Ausübung gerichteten Meinungen, Interessen und Bestrebungen hervorzubringen und zu sammeln, sie gestaltend zu formen und geltend zu machen; ebenso Hans H. Klein, a.a.O. (Fn. 23), S. 83 f. („Transformation“).

49 Ebenda verdeutlicht Markus Möstl: „Die zu Kontrollierenden (die Parteien) sollen sich nicht selbst kontrollieren (als Medienbetreiber), die zu Vermittelnden nicht selbst zu den Mittlern werden das ist der Kern der (partiellen) funktionalen Unvereinbarkeit von Parteien und Medien."

50 Vgl. BVerfGE 73, S. 118, S. 159 f., S. 171. 
gefährdet, dass vereinzelt Parteien Kapitalbeteiligungen an Rundfunkunternehmen besäßen, ohne auf die Programmgestaltung Einfluss zu nehmen. ${ }^{51}$

Jedoch werden die Medienfreiheiten nicht allein im Individualinteresse gewährleistet, sondern stehen zugleich im Dienst einer „demokratiestaatlichen Funktion“52, einer „öffentlichen Aufgabe“ "53. Die Medien stellen in der repräsentativen Demokratie ein „ständiges Verbindungs- und Kontrollorgan zwischen dem Volk und seinen gewählten Vertretern in Parlament und Regierung" 54 dar. Also enthalten die Medienfreiheiten des Art. 5 Abs. 1 Satz 2 GG neben dem Abwehrrecht ein objektives Prinzip der Gesamtrechtsordnung, das den Gesetzgeber verpflichtet, sie so auszugestalten, dass die Medien ihrer demokratiestaatlichen Funktion und ihrer öffentlichen Aufgabe gerecht werden können. ${ }^{55}$

Hierfür ist eine staatsunabhängige Stellung und Finanzierung der Medien Voraussetzung, denn die Mehrheitsparteien streben nach Machterhalt. Sie sind naturgemäß nicht an der Aufdeckung von Missständen interessiert. Außerdem wollen sie den Eindruck erwecken, dass ihr Parteiprogramm die Mehrheitsmeinung der Bürger abbildet. Hiernach wäre eine gleichsam selbsterzeugte Legitimation ihrer Macht gegeben, wenn die Parteien selbst mitbestimmten, an welchen Maßstäben sie gemessen werden. Wenn Parteien und den von ihnen abhängigen Unternehmen, Personen und Vereinigungen eine Erlaubnis zur Veranstaltung von Rundfunk nicht erteilt werden darf, so ist das auch nach Auffassung des BVerfG im Hinblick auf den besonderen Status der Parteien nicht zu beanstanden. ${ }^{56}$ Dagegen spricht auch nicht die Grundrechtsberechtigung der Parteien, da diese zwar in erster Linie der gesellschaftlichen Sphäre zuzuordnen sind, jedoch aus dem Blickwinkel des Rundfunks als die zu Kontrollierenden im Lager des Staates stehen. ${ }^{57}$

Konkrete Gefährdungen für die Funktionsfähigkeit der Parteien ergeben sich aus dem Umstand, dass deren Beteiligungen an Medienunternehmen zwar ein effektives Mittel zur Verbreitung ihrer programmatischen Positionen sind. Sofern die parteiinduziert veröffentlichte Meinung aber als öffentliche Meinung, das heißt als Produkt des öffentlichen Diskurses, ausgegeben wird, treten die tatsächlichen Interessen der Bürger in den Hintergrund. Somit wäre einer politischen Willensbildung des Volkes von unten nach oben die Grundlage entzogen.

51 Vgl. Hendrik Reffken, a.a.O. (Fn. 2), S. $299 \mathrm{ff}$.

52 Christoph Degenhart, in: Rudolf Dolzer / Christian Waldhoff / Karin Graßhof (Hrsg.), Bonner Kommentar zum Grundgesetz, Heidelberg 2005, Art. 5 Abs. 1 und 2 Rn. 4.

53 BVerfGE 20, S. 162, S. 175 für die Presse; ebenso die abweichende Meinung in BVerfGE 31, S. 314, S. 337, S. 342 für Rundfunk- und Fernsehdarbietungen beziehungsweise die Massenkommunikationsmittel; Peter Badura, Gewährleistung der Freiheit des Rundfunks, in: Detlef Merten / Reiner Schmidt / Rupert Stettner (Hrsg.), Der Verwaltungsstaat im Wandel, München 1996, S. 1 ff., S. 8; vgl. \$ 21 Abs. 1 S. 1 und 2 LRG S-H.

54 BVerfGE 20, S. 162, S. 175 für die Presse; ähnlich BVerfGE 83, S. 238, S. 296; BVerfGE 90, S. 60, S. 87 jeweils für die Vermittlungsfunktion des Rundfunks; BVerfGE 91, S. 125, S. 134 für die Kritik- und Kontrollfunktion von Presse und Rundfunk; Markus Möstl, a.a.O. (Fn. 2), S. 107; Wolfgang Seiler, Pressekonzentration und publizistische Vielfalt nach zehn Jahren deutscher Einheit, in: AfP 2002, S. 1 ff., S. 5 zu $\$ 3$ der Landespressegesetze (Konsensfunktion, Informationsfunktion, Vermittlungsfunktion).

55 BVerfGE 7, S. 198, S. 204 f.; BVerfGE 57, S. 295, S. 319 f.; BVerfGE 74, S. 297, S. 323.

56 Vgl. BVerfGE 73, S. 118, S. 190.

57 So auch Markus Möstl, Fusionskontrolle im Pressewesen: Gefahr für die Meinungsvielfalt, in: Orientierungen zur Wirtschafts- und Gesellschaftspolitik 2004, H. 3, S. 74 ff., S. 76. 


\section{Modifikation der Medienfreiheiten durch Art. 21 GG}

Soweit die Freiheit der Parteien über die grundrechtlichen Freiheiten gewährleistet wird, da weder Art. 21 GG insgesamt noch eine seiner Einzelgewährleistungen Grundrechtscharakter aufweisen, dürfen die oben genannten Grundrechte lediglich in Verbindung mit Art. 21 GG unter Beachtung seiner modifizierenden Wirkung zur Anwendung gelangen. ${ }^{58}$ Entsprechende Einschränkungen sollen sicherstellen, dass die Parteien in der Lage sind, ihren grundgesetzlichen Mitwirkungsauftrag zu erfüllen. Folglich stehen die Grundrechte den Parteien lediglich in ihrem durch Art. 21 Abs. 1 Satz 1 GG definierten Aufgaben- und Funktionskreis zur Verfügung. ${ }^{59}$ Gegen die Grundrechtsqualität des Art. 21 GG spricht dabei insbesondere die Stellung der Vorschrift im zweiten Abschnitt des Grundgesetzes („Der Bund und die Länder“) und nicht im ersten Abschnitt („Die Grundrechte“) und die in Art. 93 Abs. 1 Nr. 4a GG unterbliebene Gleichstellung mit den Grundrechten. ${ }^{60}$

\section{Einwände gegen eine Beteiligungsbeschränkung}

Sofern die Gegner einer Einschränkung der Medienbeteiligungen politischer Parteien argumentieren, dass eine konkrete Gefahr nicht gegeben sei, ist dem entgegenzuhalten, dass nicht recht einzusehen ist, warum der Verfassungsstaat sich auf die polizeirechtliche Gefahrenschwelle berufen muss, um die erwerbswirtschaftliche Betätigung solcher Vereinigungen zu reglementieren, deren Gründungsfreiheit er in Art. 21 Abs. 1 Satz 2 GG selbst verfassungsrechtlich verbürgt, und die als „verfassungsrechtliche Institution“ anerkannt sind. ${ }^{61}$

Ferner muss sich nach dem innerparteilichen Demokratiegebot des Art. 21 Abs. 1 Satz 3 GG die Willensbildung von unten nach oben vollziehen. Gleichwohl sind dem zentral geführten Unternehmensbereich der SPD Mitwirkungsmöglichkeiten sowohl der Untergliederungen als auch der Parteimitglieder unbekannt. ${ }^{62}$ Das BVerfG erwidert, dass es auch zu einer Rückkopplung zwischen Staatsorganen und Volk durch die Parteien komme. Jedoch ist an dieser Stelle auf das Verhältnis von Partei- und Unternehmensführung zur Parteibasis

58 Axel Mauersberger, Die Freiheit der Parteien, Baden-Baden 1994, S. 30 mit Fn. 54; Martin Morlok, a.a.O. (Fn. 17), Art. 21 Rn. 49; zustimmend Hans H. Klein, a.a.O. (Fn. 10), S. 70. Vgl. zur terminologischen Anknüpfung Wilhelm Henke, a.a.O. (Fn. 15), Art. 21 Rn. 218; Wilhelm Henke, a.a.O. (Fn. 9), S. 239 („besonderes Gepräge“).

59 Vgl. zu dieser Sichtweise Art. 33 Abs. 5 GG im Verhältnis zu den Grundrechten der Dienstnehmer. Hier erweisen sich die Statuspflichten ebenfalls als Grenze des Freiheitsstatus.

60 Martin Morlok, a.a.O. (Fn. 17), Art. 21 Rn. 48; Rudolf Streinz, a.a.O. (Fn. 16), Art. 21 Abs. 1 Rn. 99; Hans H. Klein, in: Theodor Maunz / Günter Dürig, a.a.O. (Fn. 35), München 2005, Art. 21 Rn. 256; Philip Kunig, in: Ingo von Münch / Philip Kunig (Hrsg.), Grundgesetz-Kommentar, München 2001, Art. 21 Rn. 46; Walter Schmidt, Politische Parteien und andere Vereinigungen, in: NJW 1984, S. 762 ff., S. 766; anderer Ansicht Thorsten Koch, „Eckpunkte“ eines neuen Parteiengesetzes, in: ZG 2001, S. 339 ff., S. 342 f. in Bezug auf Wilhelm Henke, a.a.O. (Fn. 15), Art. 21 Rn. 216 f.; Hanns-Rudolf Lipphardt, Die Gleichheit der Parteien vor der öffentlichen Gewalt, Berlin 1975, S. 118 f., S. 693; Dimitris T. Tsatsos / Martin Morlok, Parteienrecht, Heidelberg 1982, S. 75, die von einem „echten Grundrecht“ sprechen. Grundlegend Axel Mauersberger, a.a.O. (Fn. 57), S. $12 \mathrm{ff} ., 26 \mathrm{ff}$.

61 BVerfGE 1, S. 208, S. 225; BVerfGE 44, S. 125, S. 145, ständige Rechtsprechung. Vgl. zu diesem Gedanken die abweichende Meinung in BVerfGE 108, S. 282, S. 314, S. 326 f.

62 Andreas Feser, Ein Fremdkörper in einer demokratischen Ordnung, in: Orientierungen zur Wirtschafts- und Gesellschaftspolitik 2004, H. 3, S. 65 ff., S. 68. 
abzustellen. Die Parteifunktionäre sollen in erster Linie die Mehrheitsmeinung der Parteimitglieder nach außen vertreten. Hiernach dürfen die Mitglieder nicht von der Willensbildung ausgeschlossen sein und muss ihre grundsätzliche Gleichwertigkeit gewährleistet sein. ${ }^{63}$ Bei einer erwerbswirtschaftlichen Betätigung der Parteien ist auch deren Chancengleichheit im politischen Wettbewerb verletzt, da über den Wahlerfolg der Parteien durch die Höhe der eingesetzten Finanzmittel mitentschieden wird. ${ }^{64}$ Überdies sind die erwirtschafteten Gewinne aus Unternehmenstätigkeit und Beteiligungen nicht von der Akzeptanz der Parteipolitik abhängig, so dass auch insoweit Verzerrungen im politischen Wettbewerb der Parteien auftreten. Bei den Medienbeteiligungen politischer Parteien wirkt sich dieser Umstand vor allem in den Kreisen mit regionalem Monopol aus. Insbesondere lässt sich nicht einwenden, der Gesetzgeber sei nicht verpflichtet, vorgegebene und sich aus den tatsächlichen Verhältnissen ergebende Wettbewerbschancen der Parteien auszugleichen ${ }^{65}$, denn das Angebotsmonopol, über das ein Alleinanbieter verfügt, ist in einem solchen Fall gerade dadurch gekennzeichnet, dass die Bürger gezwungen sind, eine Lokalzeitung zu abonnieren oder einen Lokalsender einzuschalten, um sich über das aktuelle Geschehen vor Ort zu informieren. Insbesondere dort muss auf die parteipolitische Unabhängigkeit der Alleinanbieter Verlass sein.

Ausnahme: Parteizeitschriften und -zeitungen, Parteirundfunksender, Parteiinternetangebote

Eine Ausnahme gilt indes für die Parteizeitschriften und -zeitungen ${ }^{66}$ oder die Internetangebote der Parteien. Sie würde auch für parteieigene Rundfunksender gelten. Bei der Herausgabe parteieigener Medien ist die Medienunternehmerschaft offengelegt und eine Manipulationsgefahr für die Bürger durch die Parteien nicht gegeben, so dass jedenfalls solche Medienbeteiligungen von Parteien verfassungsrechtlich unbedenklich sind. Konsumenten dieser Angebote sind regelmäßig die eigenen Mitglieder, obgleich sich die Angebote auch an Nichtmitglieder richten. Kein Bürger aber würde derartige Medien in Anspruch nehmen, um sich unvoreingenommen über das tagespolitische Geschehen zu informieren. Vielmehr nutzt er zu diesem Zweck die Presse, den Hörfunk oder das Fernsehen, auf deren Unabhängigkeit er sich verlässt. ${ }^{67}$ Somit unterliegen parteieigene Medien, die nach außen als solche erkennbar sind, insbesondere die traditionelle Parteipresse, dem Schutz des Art. 5 Abs. 1 Satz 2 GG in Verbindung mit Art. 21 Abs. 1 GG und Art. 19 Abs. 3 GG.

63 Rüdiger Sannwald, in: Bruno Schmidt-Bleibtreu / Hans Hofmann / Axel Hopfauf, Kommentar zum Grundgesetz, Köln 2008, Art. 21 Rn. 70.

64 Andreas Feser, Vermögensmacht und Medieneinfluss, Würzburg 2002, S. 201; ebenso Rainer Wend / Alexandra Albrecht-Baba, Saldierung im Rahmen des Parteiengesetzes, in: ZRP 2001, S. $561 \mathrm{ff} .$, S. 564.

65 So BVerfGE 8, S. 51, S. 66 f.; BVerfGE 85, S. 264, S. 297; Jörn Ipsen, Staatsrecht I, Neuwied 2006, Rn. 162.

66 Zum Beispiel „vorwärts - Sozialdemokratische Monatszeitung“, „Union - Das Magazin der CDU Deutschlands“, „elde, liberale depesche - Das Magazin der Liberalen“, „Disput“, „schrägstrich - Zeitschrift für bündnisgrüne Politik“, „Bayernkurier - Deutsche Wochenzeitung für Politik, Wirtschaft und Kultur".

67 Andreas Feser, a.a.O. (Fn. 61), S. 65. 


\subsubsection{Geringfügige mittelbare und unmittelbare Beteiligungen}

Nach Auffassung des BVerfG ist unklar, ob die Partei bei einer geringfügigen Beteiligung überhaupt Einfluss auf das jeweilige Rundfunkunternehmen ausüben könne. Dagegen lässt sich einwenden, dass sowohl die strukturelle Funktionsstörung außer Acht gelassen als auch der Streubesitz im Rundfunkbereich nicht gewürdigt worden ist. Denn eine kleine resolute Minderheit kann in einer indifferenten Gesellschaftergruppe erheblichen Einfluss ausüben. ${ }^{68}$ Beschnitten wird dadurch auch nicht eine für die Demokratie lebenswichtige öffentliche Kommunikationsmöglichkeit, da die Parteipolitik nach wie vor Gegenstand von Berichterstattung sein wird - mit dem entscheidenden Unterschied, dass sich die Partei nunmehr der Kritik und Kontrolle durch unabhängige Medien stellen muss.

Unberücksichtigt geblieben ist vom BVerfG auch der unvermeidbare Interessenkonflikt zwischen einer Landesmedienanstalt und einem privaten Rundfunkveranstalter in dem Fall, in dem eine Partei an einem Veranstalter beteiligt ist, der ein Programm verbreitet, das nach Auffassung der Anstalt Anlass zur Beanstandung gibt. Denn deren Organen dürfen durchaus Mitglieder des Bundestages oder des jeweiligen Landesparlaments angehören ${ }^{69}$, die regelmäßig auch Parteiämter wahrnehmen. Besondere Bedeutung erhält dieser Konflikt, wenn die entsprechenden Unternehmensbeteiligungen für die Parteien sogar wirtschaftlich erheblich sind.

Demgegenüber argumentierte der Niedersächsische Staatsgerichtshof wie folgt: Sei eine Partei mit zehn Prozent an einem Unternehmen A beteiligt und das Unternehmen A mit zehn Prozent an dem Unternehmen B, welches wiederum mit zehn Prozent an einem Rundfunkveranstalter $C$ beteiligt sei, dürfe nach der gleichgerichteten Vorschrift des $\$ 6$ Abs. 3 Satz 3 und 4 des Niedersächsischen Mediengesetzes (NdsMG) eine Zulassung nicht erteilt werden, obwohl die faktische, durchgerechnete Beteiligung der Partei beim Rundfunkveranstalter lediglich 0,1 Prozent betrage. Der nach dieser Lesart von $\$ 6$ Abs. 3 Satz 2 bis $4 \mathrm{NdsMG}$ sehr weitgehende Ausschluss politischer Parteien von der Veranstaltung privaten Rundfunks greife über den objektiv-rechtlichen Gewährleistungsinhalt der Rundfunkfreiheit gemäß Art. 5 Abs. 1 Satz 2 GG in Verbindung mit Art. 3 Abs. 2 der Niedersächsischen Verfassung hinaus, denn er sei durch die Ziele der Herstellung von Staatsferne und Überparteilichkeit sowie Meinungsvielfalt im privaten Rundfunk nicht gedeckt. ${ }^{70}$ Jedoch verfängt dieser Einwand nicht, da auch in dem Fall einer durchgehenden Beteiligung in Höhe von 50 Prozent lediglich zehn Beteiligungsstufen notwendig wären, um die durchgerechnete mittelbare Beteiligung auf unter 0,1 Prozent zu senken.

Ferner meint das BVerfG, dass gerade bezogen auf geringfügige Beteiligungen sich die betroffenen Unternehmen nur mit außerordentlichem Aufwand und wohl nicht mit letzter Sicherheit dagegen schützen könnten, dass, etwa bei mehrfach gestuften Beteiligungsverhältnissen, nicht doch eine mittelbare Minimalbeteiligung einer Partei vorliege. Jedoch ist

68 Arthur Engel, Wahrscheinlichkeitsrechnung und Statistik, Bd. 1, Stuttgart 1971, S. 131, in Anlehnung an Roger Penrose. Zum Beispiel setzt eine entschlossene Minderheit von fünf Personen die eigene Position in einem Gremium von 25 Personen, die im Übrigen zufällig entscheiden, mit einer Wahrscheinlichkeit von 86 Prozent durch.

69 Vgl. die Inkompatibilitätsregelung des $\$ 91$ LMG NRW.

70 So der Niedersächsische Staatsgerichtshof, a.a.O. (Fn. 4), S. 1519. Kritisch auch Günter Herrmann / Matthias Lausen, Rundfunkrecht, München 2004, \$18 Rn. 18; zustimmend Joachim Wieland, a.a.O. (Fn. 20), S. 110. 
diese Einschränkung der erwerbswirtschaftlichen Betätigungsfreiheit der Parteien wegen der Bedeutung der Funktionen von Parteien und Medien durchaus gerechtfertigt. Außerdem ist der Abruf der Unternehmensdaten im Hinblick auf das gemeinsame Registerportal der Länder auch nicht mit einem unangemessenen Aufwand verbunden. Schließlich ist darauf hinzuweisen, dass die gesellschaftsrechtliche Vertragsfreiheit diverse Ausgestaltungen ermöglicht, da das Verhältnis der Gesellschafter untereinander lediglich durch wenige zwingende gesetzliche Vorschriften determiniert wird und daher weitgehend dispositives Geset-

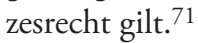

Das BVerfG führt weiter aus, dass das Prinzip der Staatsfreiheit und der Überparteilichkeit des Rundfunks schon für den Staat selbst nicht als ein striktes Trennungsgebot zu verstehen sei, sondern wegen des gesetzgeberischen Ausgestaltungsvorbehalts, der Entsendung von Vertretern in die rundfunkrechtlichen Aufsichtsgremien und der weiteren von der Rechtsprechung unbeanstandet gelassenen Einflussmöglichkeiten eher als ein System der Staatsferne zu betrachten sei. ${ }^{72}$ Jedoch ist eine Differenzierung zwischen der möglichen Beeinflussung der Programmgestaltung und der Programminhalte und der Besetzung der Organe der Landesmedienanstalten geboten. Auch das BVerfG hob in seiner Rundfunkrechtsprechung von Anfang an hervor, dass die Rundfunkfreiheit des Art. 5 Abs. 1 Satz 2 GG in erster Linie „Staatsfreiheit der Berichterstattung“ bedeute. ${ }^{73}$

Gegen eine vom Gericht zitierte Meinung im Schrifttum, wonach vereinzelt Parteien Kapitalbeteiligungen an Rundfunkunternehmen besäßen, ohne auf die Programmgestaltung Einfluss zu nehmen, lässt sich einwenden, dass die ehemalige Bundesschatzmeisterin der SPD Inge Wettig-Danielmeier selbst erklärte: „Auch dort, wo wir nur 30 oder 40 Prozent haben, kann in der Regel nichts ohne uns passieren. " 74 Natürlich nimmt die SPD Einfluss auf die grundlegenden Unternehmensentscheidungen und die Besetzung der Geschäftsführung. Dort treffen Geschäftsführer, die ohne das Vertrauen der Partei nicht in ihr Amt gekommen wären, die maßgeblichen Personalentscheidungen. Aus diesem Einfluss auf die Stellenbesetzung ergibt sich ein mittelbarer Einfluss auf die Inhalte der redaktionellen Arbeit. $^{75}$

71 Für die übliche Ausgestaltung der Medienunternehmen als GmbH oder GmbH \& Co. KG Ulrich Eisenhardt, Gesellschaftsrecht, München 2007, Rn. 675; Margarete Schuler-Harms, Medienbeteiligung politischer Parteien, in: Martin Morlok / Ulrich von Alemann / Thilo Streit, a.a.O. (Fn. 2), S. 29 ff., S. 33; Rolf Steding, Gesellschaftsrecht, Baden-Baden 1997, Rn. 48. Kritisch am Beispiel der Verlagsgesellschaft Madsack GmbH \& Co. KG beziehungsweise der Dr. Erich Madsack GmbH Andreas Feser, Der Genossen-Konzern, München 2002, S. 85.

72 Für eine Begrenzung des Einflusses der Parteien in den Aufsichtsgremien des öffentlich-rechtlichen Rundfunks Helmuth Schulze-Fielitz, a.a.O. (Fn. 27), Art. 5 Abs. 1, 2 Rn. 260; Markus Möstl, a.a.O. (Fn. 2), S. 113, Fn. 50.

73 Vgl. BVerfGE 83, S. 238, S. 322 unter Hinweis auf BVerfGE 12, S. 205, S. 262 f.

74 Zitiert nach Medien Tenor, Forschungsbericht Nr. 119 von April 2002, S. 68.

75 Andreas Feser, a.a.O. (Fn. 70), S. 66; Hans H. Klein, Parteien-Presse-Rundfunk, in: Max-Emanuel Geis / Dieter Lorenz (Hrsg.), Staat, Kirche, Verwaltung, München 2001, S. 193 ff., S. 195; Sozialdemokratische Codewörter, in: FAZ vom 12. August 2002; anders Geschäftsbericht 2002 der dd_vg., S. 30. Vgl. aber den Auszug aus dem Arbeitsvertrag mit dem Geschäftsführer der Stadtzeitschrift Szene Hamburg, zitiert nach Medien Tenor, Forschungsbericht Nr. 147 von September 2004, S. 62 ff., S. 65: „Dem Geschäftsführer ist bekannt, dass die dd_vg. der SPD nahe steht. Daraus ergibt sich für den Geschäftsführer die Verpflichtung, die sich aus diesem Verhältnis ergebende grundsätzliche Haltung ... in seiner Arbeit ... zu respektieren.“ 
Hiernach ist der Gesetzgeber gehalten, auch unterhalb der Beherrschungsschwelle Einschränkungen zu normieren, da unerheblich ist, ob im Einzelfall tatsächlich Einfluss auf die redaktionelle Arbeit nachweisbar ist, weil die Möglichkeit dazu objektiv gegeben ist und ein solcher Einfluss auch ohne ausdrückliche Weisungen möglich ist. ${ }^{76}$ Grundsätzlich reicht zwar nach dem Wortsinn ein Einzelfall für die Annahme einer strukturellen Inkompatibilität nicht aus. Sofern Bezugspunkt aber nicht der Gesamtmarkt, sondern richtigerweise die strukturelle Kollision und Spannung im Hinblick auf die Funktionen von Parteien und Medien ist, gilt schon jetzt das sich aus den objektiv-rechtlichen Gehalten des Art. 5 Abs. 1 Satz 2 GG ergebende Gebot, die Medienbeteiligungen politischer Parteien zu beschränken. Somit ist nicht entscheidend, ob eine Partei im Einzelfall Einfluss auf den Rundfunk nimmt, sondern dass die entsprechenden Veranstalter a priori in der Wahrnehmung ihrer öffentlichen Aufgabe gehemmt sind.

Durch die Pflicht zur Beendigung unzulässiger Beteiligungen werden auch die Grundrechte Dritter nicht verletzt. Bei der Vereinigungsfreiheit der Anteilseigner ist zwar problematisch, dass Art. 9 Abs. 1 GG ihnen die Befugnis einräumt, sich die Geschäftspartner für die Gründung oder den Betrieb eines Wirtschaftsunternehmens selbst auszusuchen. Sie müssten infolgedessen die zum Verkauf stehenden Anteile nach einer entsprechenden gesetzlichen Neuregelung entweder selbst erwerben oder den Verkauf an Dritte abwarten. Das dürfte indes wegen der teilweise sehr lukrativen Monopolstellungen unproblematisch möglich sein. Jedenfalls ergibt sich aus Art. 9 Abs. 1 GG weder ein Recht auf den unveränderten Fortbestand der Gesellschafter noch ein Recht darauf, dass sich der Gesellschafterbestand lediglich mit dem Einverständnis der Anteilseigner ändert. ${ }^{77}$

\subsubsection{Keine gleich geeigneten, aber mildere Mittel}

Medienbeteiligungen von Parteien zu beschränken ist auch deshalb erforderlich, weil gleich geeignete, aber mildere Mittel nicht zur Verfügung stehen. Soweit in der Diskussion über die verfassungsrechtliche Zulässigkeit von Unternehmensbeteiligungen politischer Parteien in Presse, Rundfunk und Neuen Medien das Kartellrecht oder die Offenlegung der Inhaber- und Beteiligungsverhältnisse als mögliche Alternativen genannt werden, erweisen sich diese als ungeeignet, die oben dargestellte strukturelle Inkompatibilität aufzulösen.

\section{Kartellrecht}

Bezogen auf den Pressebereich argumentierte die Parteienrechtskommission 2001 (von Wedel-Kommission), dass ein etwaiger beherrschender Einfluss von Parteien auf die Presse aufgrund von Beteiligungen im Bereich der Printmedien vorrangig mit den Mitteln und

76 Rüdiger Sannwald, a.a.O. (Fn. 62), Art. 21 Rn. 60 ff.

77 Alexandra Schindler, a.a.O. (Fn. 2), S. 210 f.; vgl. für die Kapitalgesellschaften $\$ 15$ Abs. 1 GmbHG, $\$ 68$ AktG; für die Personengesellschaften $\$ 132$ HGB, wodurch die Gesellschaft aber nicht aufgelöst wird, sondern der Gesellschafter durch seine Kündigung ausscheidet gem. $\$ 131$ Abs. 3 S. 1 Nr. 3 HGB, das gilt über den Verweis aus $\$ 161$ Abs. 2 HGB auch für die KG; etwas anderes gilt lediglich in Ausnahmefällen, $\$ 68$ Abs. $2-4$ AktG. 
nach den allgemeinen Maßstäben unter anderem des Kartellrechts einzudämmen sei. ${ }^{78}$ Jedoch ist das zur Abwehr von Missbrauchsgefahren aus einer marktbeherrschenden Stellung dienende Kartellrecht als Problemlösungsinstrument nicht geeignet, weil die Gefahren der Medienbeteiligungen von Parteien nicht in ihrer wirtschaftlichen Macht, sondern in der Konzentration von politischem und publizistischem Einfluss zu suchen sind. ${ }^{79}$

\section{Offenlegung der Inhaber- und Beteiligungsverhältnisse}

Ferner ist das BVerfG der Auffassung, dass es aufgrund der besonderen Staatsnähe der Parteien gerechtfertigt sei, auch bei geringfügiger Beteiligung an privaten Rundfunkunternehmen die Beteiligungsverhältnisse von Parteien offenzulegen. Ähnlich wie bei den Freundeskreisen in den Aufsichtsgremien des öffentlich-rechtlichen Rundfunks sei nämlich nicht auszuschließen, dass sich Interessenverbünde bei Gesellschaftern im privaten Rundfunk herausbildeten. ${ }^{80}$

Die behauptete „besondere Staatsnähe“ der Parteien impliziert typischerweise personelle Verflechtungen zwischen Parteifunktionen und Staatsämtern, zumindest bei den jeweils regierungstragenden Mehrheitsparteien. ${ }^{81}$ Auf diesem Wege eröffneten sich, trug die Landesregierung von Baden-Württemberg vor, über die Beteiligung politischer Parteien an der Veranstaltung privaten Rundfunks subtile Möglichkeiten indirekter staatlicher Einflussnahme auf die publizistische Betätigung des Rundfunks, denen es durch normative Vorkehrungen zu begegnen gelte. Folgerichtig erscheint daher zunächst der Schluss des Gerichts, dass dieser Gefahr durch ein Gebot der Veröffentlichung aller Beteiligungen begegnet werden könne.

Allerdings erklärte das Gericht nicht, in welcher Form die Offenlegung der Inhaber- und Beteiligungsverhältnisse erfolgen soll: Bei Pressebeteiligungen muss der Verleger eines periodischen Druckwerks zum Beispiel nach $\$ 7$ a Abs. 1 des Berliner Pressegesetzes (mit den am weitesten gehenden Offenlegungspflichten) ${ }^{82}$ in regelmäßigen Zeitabständen im Druckwerk die Inhaber- und Beteiligungsverhältnisse seines Verlags und seine Rechtsbeziehungen zu mit ihm verbundenen Presse- und Rundfunkunternehmen ( $\$ 15$ des Aktiengesetzes) offenlegen. Die Bekanntgabe erfolgt bei täglich oder wöchentlich erscheinenden Veröffentlichungen im Impressum der ersten Ausgabe des Kalenderhalbjahres, bei anderen periodischen Druckwerken im Impressum der ersten Ausgabe jedes Kalenderjahres. Bei Mehrheits-

78 BT-Drs. 14/6710, S. 45.

79 Petra Pohlmann, Medienbeteiligungen politischer Parteien aus kartellrechtlicher Sicht, in: Martin Morlok / Ulrich von Alemann / Thilo Streit, a.a.O. (Fn. 2), S. 51 ff., S. 53.

80 Allerdings ist darauf hinzuweisen, dass im Hinblick auf ein absolutes Beteiligungsverbot vom Gericht gerade offengelassen wurde, ob die Partei bei einer geringfügigen Beteiligung überhaupt Einfluss auf das jeweilige Rundfunkunternehmen ausüben kann. Vgl. zur Offenlegung von Treuhandverhältnissen und zur effektiveren Offenlegung der mittelbaren Unternehmensbeteiligungen Miroslav Angelov / Alexander Roth, a.a.O. (Fn. 2), S. 40.

81 Angela Merkel ist zum Beispiel Bundeskanzlerin der Bundesrepublik Deutschland (seit 2005) und zugleich Bundesvorsitzende der CDU (seit 2000). Frank-Walter Steinmeier ist Bundesminister des Auswärtigen (seit 2005), Vizekanzler der Bundesrepublik Deutschland (seit 2007) und zugleich kommissarischer Bundesvorsitzender der SPD (seit 2008).

82 Vgl. auch Art. 8 Abs. 3 BayPG, $\$ 9$ BbgPG, $\$ 5$ Abs. 2, 3 HessPG, $\$ 7$ Abs. 4 LPG M-V, $\$ 9$ Abs. 4 LMG R-P, $\$ 8$ SächsPG, $\$ 7$ Abs. 4 LPG S-H und $\$ 8$ ThürPG. 
beteiligungen von Parteien an Fernsehunternehmen ist zum Beispiel eine Einblendung der Firmenmarke in einer Bildschirmecke denkbar. Was aber soll geschehen, wenn anstelle einer Mehrheits- mehrere Minderheitsbeteiligungen gegeben sind oder wenn Parteien und andere Anteilseigner sogar gleichbehandelt werden sollen?

Gerade im Rundfunkbereich stellen sich erhebliche Praktikabilitätsprobleme, zumal der Hörer nicht imstande ist, die redaktionellen Beiträge und die Informationen über die Inhaber- und Beteiligungsverhältnisse gleichzeitig zu rezipieren. Ferner ist ein entsprechender Hinweis im Impressum desjenigen Druckwerkes ungeeignet, dessen Herausgeber auch privater Rundfunkveranstalter ist ${ }^{83}$, da der Empfängerkreis regelmäßig nicht identisch sein dürfte. Abgesehen davon dürfte der Durchschnittsleser das Impressum ohnehin nicht zur Kenntnis nehmen.

Auch die Pflicht zur öffentlichen Rechenschaftslegung gemäß $\$ \$ 23$ ff. PartG ist zur Beseitigung der strukturellen Funktionsstörung ungeeignet. Sinn und Zweck dieser Vorschriften ist nicht die Bewältigung solcher Gefahren, die von Medienbeteiligungen der Parteien für die Freiheit und Funktionsfähigkeit der Medien, sondern die von Unternehmensbeteiligungen der Parteien für ihre eigene Freiheit und Funktionsfähigkeit ausgehen. ${ }^{84}$ Die Verteilung der Rechenschaftsberichte durch den Bundestagspräsidenten als Bundestagsdrucksache nach $\$ 23$ Abs. 2 Satz 3 PartG gibt zwar sowohl Fachleuten als auch interessierten Journalisten und der politischen Konkurrenz die Möglichkeit, die Finanzierungsquellen der Parteien gründlich zu überprüfen und auch anderweitig der Öffentlichkeit zugänglich zu machen ${ }^{85}$, jedoch ist problematisch, dass der Vorstand einer Partei gemäß $₫ 23$ Abs. 1 Satz 1 PartG lediglich zum Ende eines Kalenderjahres (Rechnungsjahr) in einem Rechenschaftsbericht wahrheitsgemäß und nach bestem Wissen und Gewissen öffentlich Rechenschaft zu geben hat, so dass die Leser Berichte und Kommentare regelmäßig im Vorgang ohne entsprechendes Sonderwissen rezipieren dürften. Überdies liegt auf der Hand, dass gerade diejenigen Medien, die eine Parteibeteiligung in der Vergangenheit nicht angegeben haben, weiter schweigen werden.

\subsubsection{Absolutes Beteiligungsverbot}

Das BVerfG ist der Auffassung, das absolute Verbot für Parteien, sich an privaten Rundfunkveranstaltern zu beteiligen, sei keine zulässige gesetzgeberische Ausgestaltung der Rundfunkfreiheit. Ein Beitrag des absoluten Beteiligungsverbotes zur Sicherung der Meinungsvielfalt und Verwirklichung der Staatsfreiheit in dem vom Gericht verstandenen Sinn sei kaum feststellbar und allenfalls äußerst gering. Es sei weder von den am Verfahren Beteiligten vorgetragen noch sonst ersichtlich, dass auch Minderheitsbeteiligungen ohne Möglichkeiten zu bestimmendem Einfluss eine Gefährdung der Meinungsvielfalt im Rundfunk bewirken könnten.

83 Wenig einleuchtend erscheint, dem Leser die tatsächlichen Inhaber- und Beteiligungsverhältnisse vorenthalten zu wollen, weil ihm dadurch die Möglichkeit an die Hand gegeben würde, sein Konsumverhalten zu überdenken; so aber Martin Morlok, HessLT-Ausschussvorlage INA/16/40, S. 66.

84 So ausdrücklich Markus Möstl, a.a.O. (Fn. 2), S. 112.

85 Miroslav Angelov, a.a.O. (Fn. 2), S. 298. 
Allerdings dürften die vorgetragenen Argumente den Schluss des Gerichts nicht tragen, obgleich die Entscheidung zu dieser Frage im Ergebnis richtig ist. Unzutreffend ist etwa die Behauptung, dass bei einer die Beteiligung von Parteien an privaten Rundfunkunternehmen beschränkenden Regelung zu berücksichtigen sei, dass insbesondere die SPD dem Auftrag, bei der politischen Willensbildung des Volkes mitzuwirken, traditionell durch Beteiligungen an Presseunternehmen nachgekommen sei. Solche Beteiligungen umfassten heute in aller Regel auch mittelbare Beteiligungen an Rundfunkunternehmen. Deren vollständiger Ausschluss führe deshalb dazu, dass diese Beteiligungen nur in Verbindung mit Beteiligungen an Presseunternehmen aufgegeben werden könnten. Jedoch verfängt der Hinweis auf die geschichtliche Entwicklung der Medienbeteiligungen der SPD ${ }^{86}$ wegen des großen Bruchs in der Unternehmensstrategie nicht. Die Abkehr von der Parteipresse im engeren Sinne und die Hinwendung zu verdeckten Minderheitsbeteiligungen stellen einen Paradigmenwechsel dar, der einer Berufung auf die Vergangenheit im Wege steht. Ferner demonstrieren die Beispiele anderer Parteien, dass eine erfolgreiche Mitwirkung an der politischen Willensbildung des Volkes im Sinne des Art. 21 Abs. 1 Satz 1 GG auch ohne solche Beteiligungen möglich ist. Schließlich zeigt der in die Kritik geratene Erwerb der „Frankfurter Rundschau“ ${ }^{\text {87 }}$, dass die Unternehmenspolitik der dd_vg. durchaus auch andere Ziele verfolgt.

Die vom BVerfG weiter angesprochenen Praktikabilitätsprobleme im Hinblick auf die Bewerber mit Parteibeteiligung lassen sich durch die Einziehung einer Bagatellgrenze lösen. Da Beteiligungen in Höhe von rund 90 Prozent aus gesellschaftsrechtlicher Sicht einem vollständigen Besitz des Unternehmens gleichstehen, weil die Ausübung von Minderheitsrechten der Mitgesellschafter insoweit unmöglich ist ${ }^{88}$, scheint eine Grenze in Höhe von zehn Prozent angemessen.

\section{Fazit: Änderung des Parteiengesetzes nötig}

Aufgrund einer strukturellen Inkompatibilität der demokratiestaatlichen Funktionen von Parteien und Medien sind jedenfalls solche Medienbeteiligungen politischer Parteien verfassungsrechtlich unzulässig, durch die eine Partei auf ein anderes Unternehmen unmittelbar oder mittelbar einen beherrschenden Einfluss ausüben kann. Überdies ist - entgegen der Auffassung des Bundesverfassungsgerichts - auch ein Ausschluss geringfügiger mittelbarer und unmittelbarer Beteiligungen angezeigt, sofern eine Bagatellgrenze nicht unterschritten wird und die redaktionelle Arbeit objektiv einem politischen Einflusspotential ausgesetzt ist. Dabei dürfte der strukturellen Funktionsstörung - entgegen der Auffassung des Niedersächsischen Staatsgerichtshofs - durch eine Beschränkung der Beteiligung einer Partei an einem Medienunternehmen auf zehn Prozent auf allen Beteiligungsstufen abzuhelfen sein. Hierbei ist darauf zu achten, dass zur Abwendung von Vermögensschäden zu Lasten der Parteien ausreichende Übergangsfristen normiert werden. Eine solche Beschränkung der Unternehmensbeteiligungen von Parteien in Presse, Rundfunk und Neuen Medien ist

86 Dazu Friedhelm Boll, Entwicklung der Medienbeteiligung politischer Parteien am Beispiel der SPD, in: Martin Morlok / Ulrich von Alemann / Thilo Streit, a.a.O. (Fn. 2), S. 15 ff.

87 Vgl. Aufstand der Abhängigen, in: Die Welt vom 4. August 2004.

88 Vgl. zur Berufung einer Versammlung $\$ 50$ GmbHG. 
verfassungsrechtlich nicht nur zulässig, sondern auch geboten, weil die Grundrechte nicht ausschließlich abwehrrechtlich zu interpretieren sind, sondern auch staatliche Schutzpflichten erzeugen. Bislang wurden zum Schutz der Medienfreiheiten des Art. 5 Abs. 1 Satz 2 GG geeignete Maßnahmen nicht ergriffen, so dass das Untermaßverbot verletzt ist. Da Hauptzweck der angeregten gesetzlichen Neuregelung die Beschränkung der allgemeinen Parteienfreiheit ist, ist eine Änderung des Parteiengesetzes erforderlich.

\title{
Das rechtliche Ende politischer Parteien: Auflösung und Verschmelzung
}

\author{
Martin Limpert ${ }^{*}$
}

Das Parteiengesetz kennt vier Formen des Erlöschens von politischen Parteien: die Auflösung als Selbstauflösung, als Zwangsauflösung in einem Parteiverbotsverfahren und als Auflösung kraft Gesetzes sowie die Verschmelzung. Für die Konstellationen der Selbstauflösung, der Zwangsauflösung und der Verschmelzung gibt es in jüngster Zeit konkrete Fälle im Parteiensystem der Bundesrepublik Deutschland. Die Partei Die Grauen - Graue Panther (Kurzbezeichnung: Graue) hat sich aufgelöst. Die Linke ist durch Verschmelzung der Parteien Die Linkspartei.PDS und Wahlalternative Arbeit und soziale Gerechtigkeit (WASG) entstanden. Gegen die Nationaldemokratische Partei Deutschlands (NPD) ist ein Verbotsverfahren vor dem Bundesverfassungsgericht (BVerfG) eingeleitet worden, das wegen eines nicht behebbaren Verfahrenshindernisses eingestellt wurde.

\section{Auflösung}

\subsection{Selbstauflösung der Partei Graue}

Die Grauen befinden sich derzeit vermögensrechtlich in Liquidation. Ein Delegiertenparteitag beschloss am 1. März 2008 mit Mehrheit (42 von 57 der Stimmberechtigten) die Auflösung der Partei. ${ }^{1}$ Die am 25. März 2008 vorgenommene Auszählung der Urabstimmung ergab, dass von den 417 teilnehmenden Parteimitgliedern 342 für die Selbstauflösung gestimmt hatten. ${ }^{2}$ Die nach $\$ 17$ Abs. 1 der Satzung der Partei notwendige Zweidrittelmehrheit war damit erreicht. Am 29. März 2008 ist ein Auflösungsprotokoll verfasst und dem Bundeswahlleiter zugeleitet worden. Bereits am 25. März hatte der Bundesvorstand der Grauen ihren bisherigen ersten stellvertretenden Bundesvorsitzenden zum Liquidator bestellt.

Am 1. März 2008 ist in Berlin eine Partei unter dem Namen „Die Grauen - Generationspartei“ gegründet worden, die sich als Nachfolgerin der Partei Graue versteht, wie

\footnotetext{
Der Autor, Angehöriger der Verwaltung des Deutschen Bundestags, gibt in dem Beitrag ausschließlich seine persönliche Ansicht wieder.

1 Vgl. Meldung des ddp vom 2. März 2008.

2 Vgl. Meldung der dpa vom 25. März 2008.
} 